JOURNAL OF

FUNCTION SPACES AND APPLICATIONS

Volume 8, Number 1 (2010), 87-102
(C) 2010, Scientific Horizon http://www.jfsa.net

\title{
Summability of a Tchebysheff system of functions
}

\author{
Z.T. Abdikalikova ${ }^{1}$ and A.A. Kalybay \\ (Communicated by Pankaj Jain)
}

2000 Mathematics Subject Classification. 26D10, 41A30.

Keywords and phrases. Tchebysheff systems, lower and upper estimates, summability.

Abstract. We consider a special type of Tchebysheff systems of functions $\left\{u_{i}(\cdot)\right\}_{i=0}^{n}$ and $\left\{v_{i}(\cdot)\right\}_{i=0}^{n}$ defined on the intervals $(0,1]$ and $[1,+\infty)$, respectively, such that

$$
u_{i}(t)=t^{\alpha_{0}} \int_{t}^{1} t_{1}^{\alpha_{1}} \int_{t_{1}}^{1} t_{2}^{\alpha_{2}} \ldots \int_{t_{i-1}}^{1} t_{i}^{\alpha_{i}} d t_{i} d t_{i-1} \ldots d t_{1}
$$

and

$$
v_{i}(t)=t^{\beta_{0}} \int_{1}^{t} t_{1}^{\beta_{1}} \int_{1}^{t_{1}} t_{2}^{\beta_{2}} \ldots \int_{1}^{t_{i-1}} t_{i}^{\beta_{i}} d t_{i} d t_{i-1} \ldots d t_{1} .
$$

We find necessary and sufficient conditions under which functions from the investigated systems belong to the corresponding Lebesgue spaces $L_{p}(0,1)$ and $L_{p}(1,+\infty)$. In order to prove the main results we obtain lower and upper estimates of these functions that are of independent interest.

\footnotetext{
${ }^{1}$ This research work was supported by the INTAS grant Ref. Nr 05-1000008-8157.
} 


\section{Introduction}

Let $\alpha_{i}$ and $\beta_{i}, i=0,1, \ldots, n$, be real numbers. On the intervals $(0,1]$ and $[1,+\infty)$, respectively, we consider the following systems of functions:

$$
\left\{\begin{array}{l}
u_{0}(t)=t^{\alpha_{0}} \\
u_{1}(t)=t^{\alpha_{0}} \int_{t}^{1} t_{1}^{\alpha_{1}} d t_{1} \\
u_{2}(t)=t^{\alpha_{0}} \int_{t}^{1} t_{1}^{\alpha_{1}} \int_{t_{1}}^{1} t_{2}^{\alpha_{2}} d t_{2} d t_{1} \\
\ldots \\
u_{n}(t)=t^{\alpha_{0}} \int_{t}^{1} t_{1}^{\alpha_{1}} \int_{t_{1}}^{1} t_{2}^{\alpha_{2}} \ldots \int_{t_{n-1}}^{1} t_{n}^{\alpha_{n}} d t_{n} d t_{n-1} \ldots d t_{1}
\end{array}\right.
$$

and

$$
\left\{\begin{array}{l}
v_{0}(t)=t^{\beta_{0}} \\
v_{1}(t)=t^{\beta_{0}} \int_{1}^{t} t_{1}^{\beta_{1}} d t_{1} \\
v_{2}(t)=t^{\beta_{0}} \int_{1}^{t} t_{1}^{\beta_{1}} \int_{1}^{t_{1}} t_{2}^{\beta_{2}} d t_{2} d t_{1} \\
\ldots \\
v_{n}(t)=t^{\beta_{0}} \int_{1}^{t} t_{1}^{\beta_{1}} \int_{1}^{t_{1}} t_{2}^{\beta_{2}} \ldots \int_{1}^{t_{n-1}} t_{n}^{\beta_{n}} d t_{n} d t_{n-1} \ldots d t_{1} .
\end{array}\right.
$$

These functional systems $\left\{u_{i}(\cdot)\right\}_{i=0}^{n}$ and $\left\{v_{i}(\cdot)\right\}_{i=0}^{n}$ form Tchebysheff systems or $\mathrm{T}-$ systems on the intervals where they are defined. Moreover, the second system $\left\{v_{i}(\cdot)\right\}_{i=0}^{n}$ is an extended complete Tchebysheff system or ECT - system.

$\mathrm{T}$ - systems are very important in different areas of analysis, the theory of differential equations and statistics, e.g., in the theory of approximation (interpolation methods, cubature formulas), in boundary-value problems and problems with oscillation properties of zeros of the solutions of differential equations, and in the theory of statistical inequalities. In the monographs [1] and [2] we can find an almost complete presentation of applications of Tchebysheff systems.

Let us illustrate at least one classical problem with a solution given in terms of a Tchebysheff system.

Problem definition. Let $P_{n}(\cdot)$ be a generalized polynomial $P_{n}(\cdot)=$ $\sum_{i=0}^{n} c_{i} u_{i}(\cdot)$, where $\left\{u_{i}(\cdot)\right\}_{i=0}^{n}$ are real functions from a function space $\Phi[a, b]$.

In the case when $\Phi[a, b]$ is a space of continuous functions and $u(\cdot) \in$ 
$\Phi[a, b]$, it is required to find a polynomial $P_{n}^{0}(\cdot)$ such that $\left\|u-P_{n}^{0}\right\|=$ $\inf _{P_{n}}\left\|u-P_{n}\right\|$. This polynomial is called the polynomial of the best uniform approximation.

Haar Theorem. For any continuous function $u(\cdot)$ there exists a unique polynomial of the best uniform approximation if and only if the system of functions $\left\{u_{i}(\cdot)\right\}_{i=0}^{n}$ is a Tchebysheff system on $[a, b]$.

In our turn we study $\mathrm{T}$ - systems $\left\{u_{i}(\cdot)\right\}_{i=0}^{n}$ and $\left\{v_{i}(\cdot)\right\}_{i=0}^{n}$ in connection with problems of correct posing of some boundary-value problems for $(n+1)$ :th order differential equations, which have singularities at zero and infinity. In the case when a solution of the differential equation and its derivatives do not have traces at these singular points, we consider the following generalized conditions: at zero

$$
\lim _{t \rightarrow 0} D_{i} u(t)=D_{i} u(0), i=0,1, \ldots, n,
$$

and at infinity

$$
\lim _{t \rightarrow+\infty} D_{i} u(t)=D_{i} u(\infty), i=0,1, \ldots, n,
$$

where

$$
D_{0} u(t)=\frac{u(t)}{t^{\alpha_{0}}}, D_{i} u(t)=\frac{1}{t^{\alpha_{i}}} \frac{d}{d t} D_{i-1} u(t), i=1,2, \ldots, n .
$$

It is obvious that the introduced differential operator (1.3) and the systems $\left\{u_{i}(\cdot)\right\}_{i=0}^{n}$ and $\left\{v_{i}(\cdot)\right\}_{i=0}^{n}$ have the following close connection: these two systems (here for the second system consider $\alpha$ instead of $\beta$ ) are the fundamental systems of the solutions of the equation:

$$
D_{n+1} u(t)=0 .
$$

In order to be able to solve the boundary-value problems for equations with singularities at zero and infinity, we are faced with the problem to find conditions under which the functions from (1.1) and (1.2) belong to the Lebesgue space $L_{p}(I)$, where $I=(0,1)$ and $I=(1,+\infty)$, respectively, with the norm:

$$
\|u\|_{L_{p}}:=\left(\int_{I}|u(t)|^{p} d t\right)^{\frac{1}{p}}, 1 \leq p<\infty .
$$


In this paper we present a complete solution of this problem. In particular, our result can be useful to solve the approximation problem given above or in many other applications of Tchebysheff systems.

If in the system (1.2) we make a change variables $t=\frac{1}{x}$, then we get the system of functions $\left\{\widetilde{v}_{i}(x)\right\}_{i=0}^{n}$, which are defined on the interval $(0,1]$ and have the same forms as functions from $\left\{u_{i}(t)\right\}_{i=0}^{n}$, i.e.:

(1.4)

$$
\left\{\begin{array}{l}
\widetilde{v}_{0}(x)=x^{-\beta_{0}} \\
\widetilde{v}_{1}(x)=x^{-\beta_{0}} \int_{x}^{1} x_{1}^{-\left(\beta_{1}+2\right)} d x_{1} \\
\widetilde{v}_{2}(x)=x^{-\beta_{0}} \int_{x}^{1} x_{1}^{-\left(\beta_{1}+2\right)} \int_{x_{1}}^{1} x_{2}^{-\left(\beta_{2}+2\right)} d x_{2} d x_{1} \\
\ldots \\
\widetilde{v}_{n}(x)=x^{-\beta_{0}} \int_{x}^{1} x_{1}^{-\left(\beta_{1}+2\right)} \int_{x_{1}}^{1} x_{2}^{-\left(\beta_{2}+2\right)} \ldots \int_{x_{n-1}}^{1} x_{n}^{-\left(\beta_{n}+2\right)} d x_{n} d x_{n-1} \ldots d x_{1} .
\end{array}\right.
$$

Therefore, we consider the system (1.1) as the main object of our investigation.

The paper consists of three sections. It is organized as follows: In Section 2 we state and prove our main result (Theorem 1) concerning a characterization of the functions in the studied system, which belong to the space $L_{p}(0,1)$. Moreover, we present some lemmas concerning lower and upper estimates of functions from the investigated system. These lemmas are of independent interest but also necessary for the proof of main theorem. Finally, in Section 3 we present and discuss some further remarks and results.

Conventions. Here and in the sequel we suppose that $\sum_{j=i}^{k}=0$ if $i>k$.

The symbol $X \ll Y$ means $X \leq c Y$ with some constant $c>0$.

\section{The main result}

Our main result reads:

Theorem 1. The functions $u_{i}, i=0,1, \ldots, n$, from the system (1.1) belong to $L_{p}(0,1), 1 \leq p<\infty$, if and only if

$$
\min _{0 \leq s \leq i}\left(\alpha_{0}+\sum_{j=1}^{s}\left(\alpha_{j}+1\right)\right)>-\frac{1}{p}
$$

Remark 1. A corresponding result for the functions $v_{i}, i=0,1, \ldots, n$, from the system (1.2) is given in the next Section (see Theorem 2). 
For the proof of Theorem 1 we need two Lemmas of independent interest.

Assume that $i_{0}=\min _{k \in M_{i}} k$, where

$$
M_{i}=\left\{k: 0 \leq k \leq i, \alpha_{0}+\sum_{j=1}^{k}\left(\alpha_{j}+1\right)=\min _{0 \leq s \leq i}\left(\alpha_{0}+\sum_{j=1}^{s}\left(\alpha_{j}+1\right)\right)\right\} .
$$

Lemma 1. For the functions $u_{i}, i=1,2, \ldots, n$, from the system (1.1) and for all $0<\delta<1$ there exists $0<\delta_{1} \leq \delta$ such that for any $t \in\left(0, \delta_{1}\right]$ the following estimate

$$
c_{i}(\delta) t^{\min _{0 \leq i}\left(\alpha_{0}+\sum_{j=1}^{s}\left(\alpha_{j}+1\right)\right)} \leq u_{i}(t)
$$

holds, where $c_{i}(\delta) \rightarrow 0$ when $\delta \rightarrow 1, i=1,2, \ldots, n$.

Proof. It is obvious that $0 \leq i_{0} \leq i$. We divide the proof into three cases: (1) $i_{0}=i,(2) 0<i_{0}<i$, and (3) $i_{0}=0$. Let $t \in(0,1]$.

Case 1. $i_{0}=i$. In this case, we have

$$
u_{i}(t)=u_{i_{0}}(t)=t^{\alpha_{0}} \int_{t}^{1} t_{1}^{\alpha_{1}} \int_{t_{1}}^{1} t_{2}^{\alpha_{2}} \ldots \int_{t_{i_{0}-1}}^{1} t_{i_{0}}^{\alpha_{i_{0}}} d t_{i_{0}} d t_{i_{0}-1} \ldots d t_{1} .
$$

By changing the order of integration, we obtain that

$$
u_{i}(t)=u_{i_{0}}(t)=t^{\alpha_{0}} \int_{t}^{1} t_{i_{0}}^{\alpha_{i_{0}}} \int_{t}^{t_{i_{0}}} t_{i_{0}-1}^{\alpha_{i_{0}}-1} \ldots \int_{t}^{t_{2}} t_{1}^{\alpha_{1}} d t_{1} d t_{2} \ldots d t_{i_{0}} .
$$

Moreover, by changing variables $t_{j}=t \tau_{j}, j=1,2, \ldots, i_{0}$, in the obtained integrals, we have that

$$
u_{i}(t)=u_{i_{0}}(t)=t^{\alpha_{0}+\sum_{j=1}^{i_{0}}\left(\alpha_{j}+1\right)} \int_{1}^{1 / t} \tau_{i_{0}}^{\alpha_{i_{0}}} \int_{1}^{\tau_{i_{0}}} \tau_{i_{0}-1}^{\alpha_{i_{0}}-1} \ldots \int_{1}^{\tau_{2}} \tau_{1}^{\alpha_{1}} d \tau_{1} d \tau_{2} \ldots d \tau_{i_{0}} .
$$

It is easy to see that the function

$$
\int_{1}^{1 / t} \tau_{i_{0}}^{\alpha_{i_{0}}} \int_{1}^{\tau_{i_{0}}} \tau_{i_{0}-1}^{\alpha_{i_{0}-1}} \ldots \int_{1}^{\tau_{2}} \tau_{1}^{\alpha_{1}} d \tau_{1} d \tau_{2} \ldots d \tau_{i_{0}}
$$


is non-increasing with respect to the variable $t, 0<t<1$. Hence, for any $t \in(0, \delta]$, where $0<\delta<1$, we get that

$\int_{1}^{1 / t} \tau_{i_{0}}^{\alpha_{i_{0}}} \int_{1}^{\tau_{i_{0}}} \tau_{i_{0}-1}^{\alpha_{i_{0}-1}} \ldots \int_{1}^{\tau_{2}} \tau_{1}^{\alpha_{1}} d \tau_{1} d \tau_{2} \ldots d \tau_{i_{0}} \geq \int_{1}^{1 / \delta} \tau_{i_{0}}^{\alpha_{i_{0}}} \int_{1}^{\tau_{i_{0}}} \tau_{i_{0}-1}^{\alpha_{i_{0}-1}} \ldots \int_{1}^{\tau_{2}} \tau_{1}^{\alpha_{1}} d \tau_{1} d \tau_{2} \ldots d \tau_{i_{0}}$.

By denoting the right-hand side of the last inequality by $c_{i}^{(1)}(\delta)$, from $(2.2)$ and $(2.3)$ for any $t \in(0, \delta]$ we obtain that

$$
u_{i}(t)=u_{i_{0}}(t) \geq c_{i}^{(1)}(\delta) t^{\alpha_{0}+\sum_{j=1}^{i_{0}}\left(\alpha_{j}+1\right)}=c_{i}^{(1)}(\delta) t^{\min _{0 \leq s \leq i}\left(\alpha_{0}+\sum_{j=1}^{s}\left(\alpha_{j}+1\right)\right)},
$$

where it is obvious that $c_{i}^{(1)}(\delta) \rightarrow 0$, when $\delta \rightarrow 1$.

Case $20<i_{0}<i$. For $0<t \leq \delta<1$ we have that

$$
\begin{aligned}
u_{i}(t)= & t^{\alpha_{0}} \int_{t}^{1} t_{1}^{\alpha_{1}} \int_{t_{1}}^{1} t_{2}^{\alpha_{2}} \ldots \int_{t_{i_{0}-1}}^{1} t_{i_{0}}^{\alpha_{i_{0}}} \int_{t_{i_{0}}}^{1} t_{i_{0}+1}^{\alpha_{i_{0}+1}} \ldots \int_{t_{i-1}}^{1} t_{i}^{\alpha_{i}} d t_{i} d t_{i-1} \ldots d t_{1} \\
\geq & t^{\alpha_{0}} \int_{t}^{\delta} t_{1}^{\alpha_{1}} \int_{t_{1}}^{\delta} t_{2}^{\alpha_{2}} \ldots \int_{t_{i_{0}-1}}^{\delta} t_{i_{0}}^{\alpha_{i_{0}}} d t_{i_{0}} d t_{i_{0}-1} \ldots d t_{1} \\
& \times \int_{\delta}^{1} t_{i_{0}+1}^{\alpha_{i_{0}+1}} \int_{t_{i_{0}+1}}^{1} t_{i_{0}+2}^{\alpha_{i_{0}+2}} \ldots \int_{t_{i-1}}^{1} t_{i}^{\alpha_{i}} d t_{i} d t_{i-1} \ldots d t_{i_{0}+1} .
\end{aligned}
$$

By denoting the last line by $c_{i}^{(2)}(\delta)$, from $(2.5)$ we get that

$$
u_{i}(t) \geq c_{i}^{(2)}(\delta) t^{\alpha_{0}} \int_{t}^{\delta} t_{1}^{\alpha_{1}} \int_{t_{1}}^{\delta} t_{2}^{\alpha_{2}} \ldots \int_{t_{i_{0}-1}}^{\delta} t_{i_{0}}^{\alpha_{i_{0}}} d t_{i_{0}} d t_{i_{0}-1} \ldots d t_{1}
$$

where $c_{i}^{(2)}(\delta) \rightarrow 0$, when $\delta \rightarrow 1$.

Arguing as before for the first case, in (2.6) we change the order of integration:

$$
u_{i}(t) \geq c_{i}^{(2)}(\delta) t^{\alpha_{0}} \int_{t}^{\delta} t_{i_{0}}^{\alpha_{i_{0}}} \int_{t}^{t_{i_{0}}} t_{i_{0}-1}^{\alpha_{i_{0}-1}} \ldots \int_{t}^{t_{2}} t_{1}^{\alpha_{1}} d t_{1} d t_{2} \ldots d t_{i_{0}} .
$$


Next we change variables $t_{j}=t \tau_{j}, j=1,2, \ldots, i_{0}$, in the integrals from (2.7):

(2.8) $u_{i}(t) \geq c_{i}^{(2)}(\delta) t^{\alpha_{0}+\sum_{j=1}^{i_{0}}\left(\alpha_{j}+1\right)} \int_{1}^{\delta / t} \tau_{i_{0}}^{\alpha_{i_{0}}} \int_{1}^{\tau_{i_{0}}} \tau_{i_{0}-1}^{\alpha_{i_{0}}-1} \ldots \int_{1}^{\tau_{2}} \tau_{1}^{\alpha_{1}} d \tau_{1} d \tau_{2} \ldots d \tau_{i_{0}}$.

Due to the fact that the function

$$
\int_{1}^{\delta / t} \tau_{i_{0}}^{\alpha_{i_{0}}} \int_{1}^{\tau_{i_{0}}} \tau_{i_{0}-1}^{\alpha_{i_{0}-1}} \ldots \int_{1}^{\tau_{2}} \tau_{1}^{\alpha_{1}} d \tau_{1} d \tau_{2} \ldots d \tau_{i_{0}}
$$

is non-increasing with respect to the variable $t, 0<t \leq \delta$, for any $t \in\left(0, \frac{1}{2} \delta\right]$, we have that

$\int_{1}^{\delta / t} \tau_{i_{0}}^{\alpha_{i_{0}}} \int_{1}^{\tau_{i_{0}}} \tau_{i_{0}-1}^{\alpha_{i_{0}-1}} \ldots \int_{1}^{\tau_{2}} \tau_{1}^{\alpha_{1}} d \tau_{1} d \tau_{2} \ldots d \tau_{i_{0}} \geq \int_{1}^{2} \tau_{i_{0}}^{\alpha_{i_{0}}} \int_{1}^{\tau_{i_{0}}} \tau_{i_{0}-1}^{\alpha_{i_{0}-1}} \ldots \int_{1}^{\tau_{2}} \tau_{1}^{\alpha_{1}} d \tau_{1} d \tau_{2} \ldots d \tau_{i_{0}}$.

By denoting the last integral by $c_{i}^{(3)}$, from (2.8) and (2.9) for any $t \in\left(0, \frac{1}{2} \delta\right]$ we find that

$$
u_{i}(t) \geq c_{i}^{(2)}(\delta) c_{i}^{(3)} t^{\alpha_{0}+\sum_{j=1}^{i_{0}}\left(\alpha_{j}+1\right)}=c_{i}^{(2)}(\delta) c_{i}^{(3)} t^{0 \leq \min ^{0 \leq i}}\left(\alpha_{0}+\sum_{j=1}^{s}\left(\alpha_{j}+1\right)\right),
$$

where $c_{i}^{(2)}(\delta) c_{i}^{(3)} \rightarrow 0$, when $\delta \rightarrow 1$.

$\underline{\text { Case } 3} i_{0}=0$. In this case, $\alpha_{0}=\alpha_{i_{0}}$. Hence, the function $u_{i}$ can be presented in the following form:

$$
u_{i}(t)=t^{\alpha_{i_{0}}} \int_{t}^{1} t_{i_{0}+1}^{\alpha_{i_{0}+1}} \int_{t_{i_{0}+1}}^{1} t_{i_{0}+2}^{\alpha_{i_{0}+2}} \ldots \int_{t_{i-1}}^{1} t_{i}^{\alpha_{i}} d t_{i} d t_{i-1} \ldots d t_{i_{0}+1}
$$

Arguing in the same way as in the previous cases, for $t, 0<t \leq \delta<1$, we have that

$$
\begin{aligned}
& \int_{t}^{1} t_{i_{0}+1}^{\alpha_{i_{0}+1}} \int_{t_{i_{0}+1}}^{1} t_{i_{0}+2}^{\alpha_{i_{0}+2}} \ldots \int_{t_{i-1}}^{1} t_{i}^{\alpha_{i}} d t_{i} d t_{i-1} \ldots d t_{i_{0}+1} \\
& \quad \geq \int_{\delta}^{1} t_{i_{0}+1}^{\alpha_{i_{0}+1}} \int_{t_{i_{0}+1}}^{1} t_{i_{0}+2}^{\alpha_{i_{0}+2}} \ldots \int_{t_{i-1}}^{1} t_{i}^{\alpha_{i}} d t_{i} d t_{i-1} \ldots d t_{i_{0}+1} .
\end{aligned}
$$


By denoting the last integral by $c_{i}^{(4)}(\delta)$, for any $t \in(0, \delta]$ we obtain that

$$
u_{i}(t) \geq c_{i}^{(4)}(\delta) t^{\alpha_{i_{0}}}=c_{i}^{(4)}(\delta) t^{\min _{0 \leq s \leq i}\left(\alpha_{0}+\sum_{j=1}^{s}\left(\alpha_{j}+1\right)\right)},
$$

where $c_{i}^{(4)}(\delta) \rightarrow 0$, when $\delta \rightarrow 1$.

By combining (2.4), (2.10), and (2.11) we obtain (2.1).

Lemma 2. For the functions $u_{i}, i=1,2, \ldots, n$, from the system (1.1) for any $t \in(0,1]$ the following estimate

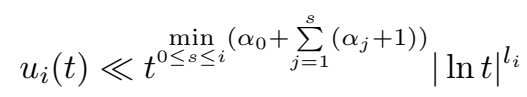

holds, where $l_{i}$ is the number of $k, i_{0}+1 \leq k \leq i$, such that $\sum_{j=i_{0}+1}^{k}\left(\alpha_{j}+1\right)=$ 0 , if $i_{0}<i$, and $l_{i}=0$ if $i_{0}=i$.

Proof. By assumption, $i_{0}$ is the minimal index such that $0 \leq i_{0}<i$ and

$$
\alpha_{0}+\sum_{j=1}^{i_{0}}\left(\alpha_{j}+1\right)=\min _{0 \leq s \leq i}\left(\alpha_{0}+\sum_{j=1}^{s}\left(\alpha_{j}+1\right)\right) .
$$

Therefore, for $i_{0}<s \leq i$ we have that

$$
\left(\alpha_{0}+\sum_{j=1}^{i_{0}}\left(\alpha_{j}+1\right)\right)-\left(\alpha_{0}+\sum_{j=1}^{s}\left(\alpha_{j}+1\right)\right)=-\sum_{j=i_{0}+1}^{s}\left(\alpha_{j}+1\right) \leq 0,
$$

and, if $i_{0}>0$, for $0 \leq s \leq i_{0}-1$ we have that

$$
\left(\alpha_{0}+\sum_{j=1}^{i_{0}}\left(\alpha_{j}+1\right)\right)-\left(\alpha_{0}+\sum_{j=1}^{s}\left(\alpha_{j}+1\right)\right)=\sum_{j=s+1}^{i_{0}}\left(\alpha_{j}+1\right)<0 .
$$

Let us again divide the proof of Lemma 2 into three cases: (1) $i_{0}=i,(2)$ $0<i_{0}<i$, and $(3) i_{0}=0$. Let $t \in(0,1]$.

Case $1 i_{0}=i$. Here $l_{i}=l_{i_{0}}=0$. According to (2.14) we have that

$$
\begin{aligned}
u_{i}(t)=u_{i_{0}}(t) & =t^{\alpha_{0}} \int_{t}^{1} t_{1}^{\alpha_{1}} \int_{t_{1}}^{1} t_{2}^{\alpha_{2}} \ldots \int_{t_{i_{0}-1}}^{1} t_{i_{0}}^{\alpha_{i_{0}}} d t_{i_{0}} d t_{i_{0}-1} \ldots d t_{1} \\
& \leq t^{\alpha_{0}} \int_{t}^{\infty} t_{1}^{\alpha_{1}} \int_{t_{1}}^{\infty} t_{2}^{\alpha_{2}} \ldots \int_{t_{i_{0}-1}}^{\infty} t_{i_{0}}^{\alpha_{i_{0}}} d t_{i_{0}} d t_{i_{0}-1} \ldots d t_{1}
\end{aligned}
$$




$$
\leq c_{i}^{(5)} t^{\alpha_{0}+\sum_{j=1}^{i_{0}}\left(\alpha_{j}+1\right)}=c_{i}^{(5)} t^{\min ^{0 \leq s \leq i}\left(\alpha_{0}+\sum_{j=1}^{s}\left(\alpha_{j}+1\right)\right)}|\ln t|^{l_{i}},
$$

where

$$
c_{i}^{(5)}=\left(\prod_{k=1}^{i_{0}}\left|\sum_{j=k}^{i_{0}}\left(\alpha_{j}+1\right)\right|\right)^{-1} .
$$

Case $20<i_{0}<i$. By changing the order of integration in the inter integral, we can present $u_{i}$ in the following form:

$$
\begin{aligned}
u_{i}(t)= & t^{\alpha_{0}} \int_{t}^{1} t_{1}^{\alpha_{1}} \int_{t_{1}}^{1} t_{2}^{\alpha_{2}} \ldots \int_{t_{i_{0}-1}}^{1} t_{i_{0}}^{\alpha_{i_{0}}} \int_{t_{i_{0}}}^{1} t_{i_{0}+1}^{\alpha_{i_{0}+1}} \ldots \int_{t_{i-1}}^{1} t_{i}^{\alpha_{i}} d t_{i} d t_{i-1} \ldots d t_{1} \\
= & t^{\alpha_{0}} \int_{t}^{1} t_{1}^{\alpha_{1}} \int_{t_{1}}^{1} t_{2}^{\alpha_{2}} \ldots \int_{t_{i_{0}-1}}^{1} t_{i_{0}}^{\alpha_{i_{0}}} \\
& \times\left(\int_{t_{i_{0}}}^{1} t_{i}^{\alpha_{i}} \int_{t_{i_{0}}}^{t_{i}} t_{i-1}^{\alpha_{i-1}} \ldots \int_{t_{i_{0}}}^{t_{i_{0}+2}} t_{i_{0}+1}^{\alpha_{i_{0}+1}} d t_{i_{0}+1} d t_{i_{0}+2} \ldots d t_{i}\right) d t_{i_{0}} d t_{i_{0}-1} \ldots d t_{1} .
\end{aligned}
$$

We separately consider the following integral:

$$
I_{\left(i, i_{0}\right)}\left(t_{i_{0}}\right)=\int_{t_{i_{0}}}^{1} t_{i}^{\alpha_{i}} \int_{t_{i_{0}}}^{t_{i}} t_{i-1}^{\alpha_{i-1}} \ldots \int_{t_{i_{0}}}^{t_{i_{0}+2}} t_{i_{0}+1}^{\alpha_{i_{0}+1}} d t_{i_{0}+1} d t_{i_{0}+2} \ldots d t_{i} .
$$

According to (2.13) for $s=i_{0}+1$ we have that $\alpha_{i_{0}+1}+1 \geq 0$. There are two possible cases: either $\alpha_{i_{0}+1}+1=0$ or $\alpha_{i_{0}+1}+1>0$. For the first case $\alpha_{i_{0}+1}+1=0$, by taking into account that $t \leq t_{i_{0}} \leq t_{i_{0}+2} \leq 1$, we get that

$$
\int_{t_{i_{0}}}^{t_{i_{0}+2}} t_{i_{0}+1}^{\alpha_{i_{0}+1}} d t_{i_{0}+1}=\int_{t_{i_{0}}}^{t_{i_{0}+2}} t_{i_{0}+1}^{-1} d t_{i_{0}+1} \leq \int_{t}^{1} t_{i_{0}+1}^{-1} d t_{i_{0}+1}=|\ln t| .
$$

This gives that

$$
I_{\left(i, i_{0}+1\right)}\left(t_{i_{0}}\right) \leq|\ln t| \int_{t_{i_{0}}}^{1} t_{i}^{\alpha_{i}} \int_{t_{i_{0}}}^{t_{i}} t_{i-1}^{\alpha_{i-1}} \ldots \int_{t_{i_{0}}}^{t_{i_{0}+3}} t_{i_{0}+2}^{\alpha_{i_{0}+2}+\alpha_{i_{0}+1}+1} d t_{i_{0}+2} d t_{i_{0}+3} \ldots d t_{i}
$$

for $0<t \leq t_{0}<1$. 
If $\alpha_{i_{0}+1}+1>0$, then

$$
\int_{t_{i_{0}}}^{t_{i_{0}+2}} t_{i_{0}+1}^{\alpha_{i_{0}+1}} d t_{i_{0}+1} \leq \int_{0}^{t_{i_{0}+2}} t_{i_{0}+1}^{\alpha_{i_{0}+1}} d t_{i_{0}+1}=\frac{1}{\alpha_{i_{0}+1}+1} t_{i_{0}+2}^{\alpha_{i_{0}+1+1}}
$$

Hence,

$$
I_{\left(i, i_{0}+1\right)}\left(t_{i_{0}}\right) \leq \frac{1}{\alpha_{i_{0}+1}+1} \int_{t_{i_{0}}}^{1} t_{i}^{\alpha_{i}} \int_{t_{i_{0}}}^{t_{i}} t_{i-1}^{\alpha_{i-1}} \ldots \int_{t_{i_{0}}}^{t_{i_{0}+3}} t_{i_{0}+2}^{\alpha_{i_{0}+2}+\alpha_{i_{0}+1}+1} d t_{i_{0}+2} d t_{i_{0}+3} \ldots d t_{i} .
$$

In (2.18) and (2.19) the integral expressions are equal, and the number of iterated integrals is one less than in (2.17). From (2.13), by continuing to reduce the number of iterated integrals, for $0<t \leq t_{i_{0}} \leq 1$, we have that

$$
I_{\left(i, i_{0}\right)}\left(t_{i_{0}}\right) \leq c_{i}^{(6)}|\ln t|^{l_{i}},
$$

where $c_{i}^{(6)}, i=0,1, \ldots, n$, depend only on $\alpha_{i}, i=0,1, \ldots, n$.

Hence, from (2.16) and (2.20) it follows that

$u_{i}(t) \leq c_{i}^{(6)}|\ln t|^{l_{i}} t^{\alpha_{0}} \int_{t}^{1} t_{1}^{\alpha_{1}} \int_{t_{1}}^{1} t_{2}^{\alpha_{2}} \ldots \int_{t_{i_{0}}-1}^{1} t_{i_{0}}^{\alpha_{i_{0}}} d t_{i_{0}} d t_{i_{0}-1} \ldots d t_{1}=c_{i}^{(6)}|\ln t|^{l_{i}} u_{i_{0}}(t)$.

In view of (2.15) this estimate yields

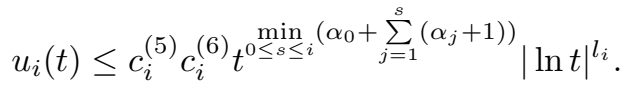

Case $3 i_{0}=0$. By changing the order of integration, the function $u_{i}$ can be presented in the following form:

$u_{i}(t)=t^{\alpha_{i}} \int_{t}^{1} t_{i}^{\alpha_{i}} \int_{t}^{t_{i}} t_{i-1}^{\alpha_{i-1}} \ldots \int_{t}^{t_{2}} t_{1}^{\alpha_{1}} d t_{1} d t_{2} \ldots d t_{i}=t^{\min ^{0 \leq s \leq i}}{ }^{\left(\alpha_{0}+\sum_{j=1}^{s}\left(\alpha_{j}+1\right)\right)} I_{(i, 0)}(t)$.

Since in this case, due to (2.13), $\sum_{j=1}^{s}\left(\alpha_{j}+1\right) \geq 0$ for all $1 \leq s \leq i$, then in the same way as in the previous case it is easy to prove that $I_{(i, 0)}(t) \leq c_{i}^{(7)}|\ln t|^{l_{i}}$, where $c_{i}^{(7)}, i=0,1, \ldots, n$, depend only on $\alpha_{i}$, $i=0,1, \ldots, n$. 
This estimate together with (2.22) give that

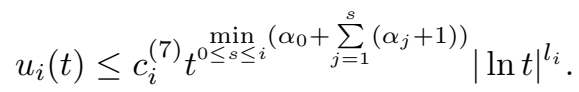

By combining (2.15), (2.21) and (2.23) we obtain (2.12).

Remark 2. The assumptions of Lemma 1 and Lemma 2 hold also for the functions $u_{0}$, since in this case $i_{0}=0$ and $l_{0}=0$, and, thus,

$$
u_{0}=t^{\alpha_{0}}=t^{\min _{0 \leq s \leq 0}\left(\alpha_{0}+\sum_{j=1}^{s}\left(\alpha_{j}+1\right)\right)}|\ln t|^{l_{0}} .
$$

Proof of Theorem 1. Let $u_{i} \in L_{p}(0,1)$. Then, due to Lemma 1 (see (2.1))

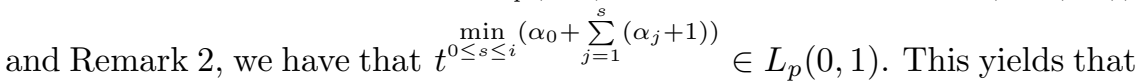

$$
\min _{0 \leq s \leq i}\left(\alpha_{0}+\sum_{j=1}^{s}\left(\alpha_{j}+1\right)\right)>-\frac{1}{p} .
$$

Now suppose that $\min _{0 \leq s \leq i}\left(\alpha_{0}+\sum_{j=1}^{s}\left(\alpha_{j}+1\right)\right)>-\frac{1}{p}$. Then, for every $i$ there exists $\delta_{i}>0$ such that

$$
\min _{0 \leq s \leq i}\left(\alpha_{0}+\sum_{j=1}^{s}\left(\alpha_{j}+1\right)\right)-\delta_{i}>-\frac{1}{p} .
$$

Moreover, according to Lemma 2 (see (2.12)) and Remark 2, it yields that

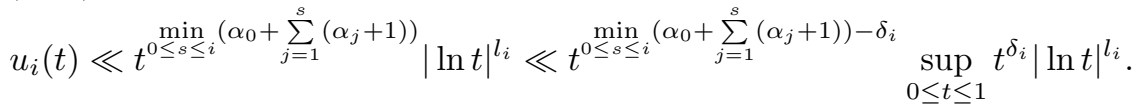

Since $\sup _{0 \leq t \leq 1} t^{\delta_{i}}|\ln t|^{l_{i}}<\infty$ for any $l_{i} \geq 0$, then from (2.25) and (2.24) we conclude that $u_{i} \in L_{p}(0,1)$.

\section{Further remarks and results}

The following Corollary follows from Lemma 1 and Lemma 2:

Corollary 1. If $\alpha_{0}+\sum_{j=1}^{m}\left(\alpha_{j}+1\right) \neq \alpha_{0}+\sum_{j=1}^{l}\left(\alpha_{j}+1\right)$ for $m \neq l$, $m, l=0,1, \ldots, n$, then for the functions $u_{i}, i=1,2, \ldots, n$, from the system 
(1.1) for $0<\delta<1$ the following estimate holds

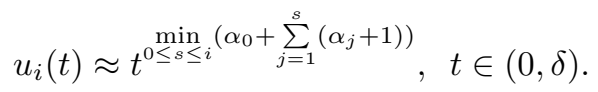

Let us now turn to the problem of summability of functions from (1.2).

Theorem 2. The functions $v_{i}, i=0,1, \ldots, n$, from the system (1.2) belong to $L_{p}(1,+\infty), 1 \leq p<\infty$, if and only if

$$
\max _{0 \leq s \leq i}\left(\beta_{0}+\sum_{j=1}^{s}\left(\beta_{j}+1\right)\right)<-\frac{1}{p} .
$$

It was mentioned above that if in (1.2) we change variables $t=\frac{1}{x}$, then we get the system (1.4) of functions defined on $(0,1]$. Moreover, these functions have the same forms as functions from (1.1). By comparing powers of functions from (1.1) and (1.4), we have that $\alpha_{0}=-\beta_{0}, \alpha_{i}=$ $-\left(\beta_{i}+2\right), i=1,2, \ldots, n$. Therefore, all conditions we introduced for (1.1) can easily be rewritten for the system (1.4). Thus,

$$
\begin{aligned}
\min _{0 \leq s \leq i}\left(\alpha_{0}+\sum_{j=1}^{s}\left(\alpha_{j}+1\right)\right) & =\min _{0 \leq s \leq i}\left(-\beta_{0}+\sum_{j=1}^{s}\left(-\left(\beta_{j}+2\right)+1\right)\right) \\
& =-\max _{0 \leq s \leq i}\left(\beta_{0}+\sum_{j=1}^{s}\left(\beta_{j}+1\right)\right) .
\end{aligned}
$$

Now, $i_{0}=\min _{k \in L_{i}} k$, where

$$
L_{i}=\left\{k: 0 \leq k \leq i, \beta_{0}+\sum_{j=1}^{k}\left(\beta_{j}+1\right)=\max _{0 \leq s \leq i}\left(\beta_{0}+\sum_{j=1}^{s}\left(\beta_{j}+1\right)\right)\right\} .
$$

Moreover, the conditions $\sum_{j=i_{0}+1}^{k}\left(\alpha_{j}+1\right)=0$ and $\sum_{j=i_{0}+1}^{k}\left(\beta_{j}+1\right)=0$ are equivalent since

$$
\sum_{j=i_{0}+1}^{k}\left(\alpha_{j}+1\right)=\sum_{j=i_{0}+1}^{k}\left(-\left(\beta_{j}+2\right)+1\right)=-\sum_{j=i_{0}+1}^{k}\left(\beta_{j}+1\right)=0 .
$$

Finally, to prove Theorem 2 we state and prove the corresponding Lemmas (to Lemmas 1 and Lemma 2).

Lemma 3. For the functions $v_{i}, i=1,2, \ldots, n$, from the system (1.2) and for all $\lambda>1$ there exists $\lambda_{1} \geq \lambda>1$ such that for any $t \in\left[\lambda_{1},+\infty\right)$ 
the estimate

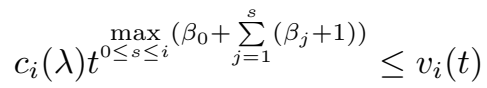

holds, where $c_{i}(\lambda) \rightarrow 0$ when $\lambda \rightarrow 1$.

Proof. In view of Lemma 1 and (3.1) for functions from (1.4) and for any $0<\delta<1$ there exists $0<\delta_{1} \leq \delta$ such that

$$
\widetilde{c}_{i}(\delta) x^{-\max _{0 \leq s \leq i}\left(\beta_{0}+\sum_{j=1}^{s}\left(\beta_{j}+1\right)\right)} \leq \widetilde{v}_{i}(x), \text { for } x \in\left(0, \delta_{1}\right],
$$

where $\tilde{c}_{i}(\delta) \rightarrow 0$ when $\delta \rightarrow 1$.

Let $\lambda=\frac{1}{\delta}, \lambda_{1}=\frac{1}{\delta_{1}}$. Since $\widetilde{v}_{i}(x)=v_{i}(t)$ for $x=\frac{1}{t}$, we can substitute them into (3.3) and then, for any $t \in\left[\lambda_{1},+\infty\right)$, we get that

$$
\widetilde{c}_{i}\left(\frac{1}{\lambda}\right)\left(\frac{1}{t}\right)^{-\max _{0 \leq s \leq i}\left(\beta_{0}+\sum_{j=1}^{s}\left(\beta_{j}+1\right)\right)} \leq v_{i}(t),
$$

where $\tilde{c}_{i}\left(\frac{1}{\lambda}\right) \rightarrow 0$ when $\lambda \rightarrow 1$, i.e. that

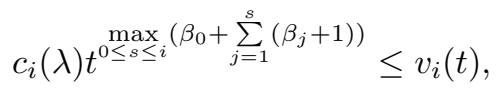

where $c_{i}(\lambda) \equiv \tilde{c}_{i}\left(\frac{1}{\lambda}\right) \rightarrow 0$ when $\lambda \rightarrow 1$.

Lemma 4. For the functions $v_{i}, i=1,2, \ldots, n$, from the system (1.2) for any $t \in[1,+\infty)$ the estimate

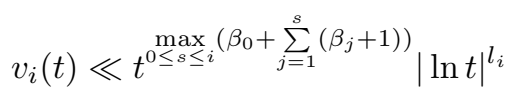

holds, where $l_{i}$ is the number of $k, i_{0}+1 \leq k \leq i$, such that $\sum_{j=i_{0}+1}^{k}\left(\beta_{j}+1\right)=$ 0 , if $i>i_{0}$, and $l_{i}=0$, if $i=i_{0}$.

Proof. Due to Lemma 2 and (3.1) for functions from (1.4) for any $x \in(0,1]$ we find that

$$
\widetilde{v}_{i}(x) \ll x^{-\max _{0 \leq s \leq i}\left(\beta_{0}+\sum_{j=1}^{s}\left(\beta_{j}+1\right)\right)}|\ln x|^{l_{i}},
$$

where, due to (3.2), $l_{i}$ is the number of $k, i_{0}+1 \leq k \leq i$, such that $\sum_{j=i_{0}+1}^{k}\left(\beta_{j}+1\right)=0$. 
In (3.4) we substitute $\widetilde{v}_{i}(x)=v_{i}(t)$ and $x=\frac{1}{t}$ and then, for any $t \in[1,+\infty)$, we get that

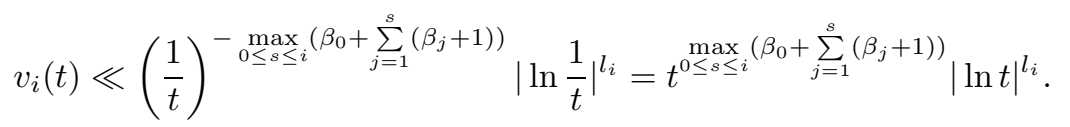

Remark 3. The assumptions of Lemma 3 and Lemma 4 hold also for the functions $v_{0}$, since in this case $i_{0}=0$ and $l_{0}=0$, and, thus, $v_{0}=t^{\beta_{0}}=t^{\max _{0 \leq s \leq 0}\left(\beta_{0}+\sum_{j=1}^{s}\left(\beta_{j}+1\right)\right)}|\ln t|^{l_{0}}$.

Proof of Theorem 2. Assume that $\max _{0 \leq s \leq i}\left(\beta_{0}+\sum_{j=1}^{s}\left(\beta_{j}+1\right)\right)<-\frac{1}{p}$. Then, for every $i$ there exists $\gamma_{i}>0$ such that

$$
\max _{0 \leq s \leq i}\left(\beta_{0}+\sum_{j=1}^{s}\left(\beta_{j}+1\right)\right)+\gamma_{i}<-\frac{1}{p} .
$$

On the other hand from Lemma 4 and Remark 3 it follows that

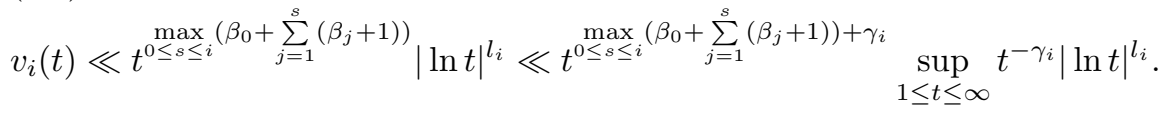

Since $\sup _{1 \leq t \leq \infty} t^{-\gamma_{i}}|\ln t|^{l_{i}}<\infty$ for any $l_{i} \geq 0$, then from (3.6) and (3.5) we have that $v_{i} \in L_{p}(1,+\infty)$.

Let now $v_{i} \in L_{p}(1,+\infty)$. Then, in view of Lemma 3 and Remark 3 , we find that

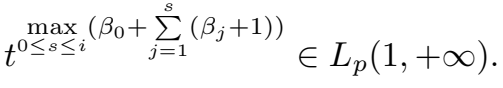

This gives that $\max _{0 \leq s \leq i}\left(\beta_{0}+\sum_{j=1}^{s}\left(\beta_{j}+1\right)\right)<-\frac{1}{p}$.

Next consider the following system:

$$
\left\{\begin{array}{l}
w_{0}(t)=t^{\alpha_{0}} \\
w_{1}(t)=t^{\alpha_{0}} \int_{t}^{1} t_{1}^{\alpha_{1}} d t_{1} \\
w_{2}(t)=t^{\alpha_{0}} \int_{t}^{1} t_{1}^{\alpha_{1}} \int_{t}^{t_{1}} t_{2}^{\alpha_{2}} d t_{2} d t_{1} \\
\ldots \\
w_{n}(t)=t^{\alpha_{0}} \int_{t}^{1} t_{1}^{\alpha_{1}} \int_{t}^{t_{1}} t_{2}^{\alpha_{2}} \ldots \int_{t}^{t_{n-1}} t_{n}^{\alpha_{n}} d t_{n} d t_{n-1} \ldots d t_{1} .
\end{array}\right.
$$


In this system the first functions $w_{0}$ and $w_{1}$ coincide with the functions $u_{0}$ and $u_{1}$ from (1.1), respectively. But, as we can see, the lower and upper integral bounds of the functions $w_{i}$ and $u_{i}, i=2,3, \ldots, n$, are correspondingly different.

By changing the order of integration in each function from (3.7), for $w_{i}$ we have that

$$
w_{i}(t)=t^{\alpha_{0}} \int_{t}^{1} t_{i}^{\alpha_{i}} \int_{t_{i}}^{1} t_{i-1}^{\alpha_{i-1}} \ldots \int_{t_{2}}^{1} t_{1}^{\alpha_{1}} d t_{1} d t_{2} \ldots d t_{i} .
$$

Now the function $w_{i}$ has the same form as the function $u_{i}$ from (1.1) with the difference in order of powers of the functions in the integrals. Hence, it is easy to formulate lower and upper estimates for $w_{i}$ and conditions of its summability to the power $p$ on the interval $(0,1)$.

Lemma 5. For the functions $w_{i}, i=1,2, \ldots, n$, from the system (3.7) and for all $0<\delta<1$ there exists $0<\delta_{1} \leq \delta$ such that for any $t \in\left(0, \delta_{1}\right]$ the estimate $c_{i}(\delta) t^{1 \leq s \leq i+1} \min _{j=s}\left(\alpha_{0}+\sum_{j=s}^{i}\left(\alpha_{j}+1\right)\right) \leq w_{i}(t)$ holds, where $c_{i}(\delta) \rightarrow 0$ when $\delta \rightarrow 1, i=1,2, \ldots, n$.

Lemma 6. For the functions $w_{i}, i=1,2, \ldots, n$, from the system (3.7) for any $t \in(0,1]$ the following estimate

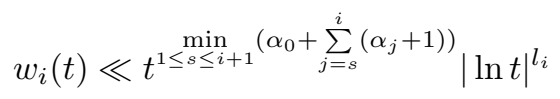

holds, where $l_{i}$ is the number of $k, 1 \leq k \leq i_{0}-1$, such that $\sum_{j=k}^{i_{0}-1}\left(\alpha_{j}+1\right)=$ 0 , when $1<i_{0}$, and $l_{i}=0$ when $i_{0}=1$.

$$
\begin{aligned}
& \text { Here } i_{0}=\max _{k \in N_{i}} k \text {, where } \\
& N_{i}=\left\{k: 1 \leq k \leq i+1, \alpha_{0}+\sum_{j=k}^{i}\left(\alpha_{j}+1\right)=\min _{1 \leq s \leq i+1}\left(\alpha_{0}+\sum_{j=s}^{i}\left(\alpha_{j}+1\right)\right)\right\} .
\end{aligned}
$$

Remark 4. The assumptions of Lemma 5 and 6 hold also for the functions $w_{0}$, since in this case $l_{0}=0$, and, thus,

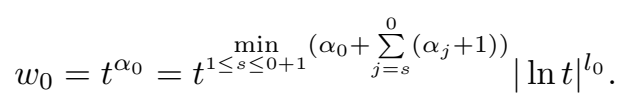


Theorem 3. The functions $w_{i}, i=0,1, \ldots, n$, from the system (3.7) belong to $L_{p}(0,1), 1 \leq p<\infty$, if and only if

$$
\min _{1 \leq s \leq i+1}\left(\alpha_{0}+\sum_{j=s}^{i}\left(\alpha_{j}+1\right)\right)>-\frac{1}{p} .
$$

Remark 5. Consider the system $\left\{y_{i}(\cdot)\right\}_{i=0}^{n}$ with $i$ :th function in the form:

$$
y_{i}(t)=t^{\beta_{0}} \int_{1}^{t} t_{1}^{\beta_{1}} \int_{t_{1}}^{t} t_{2}^{\beta_{2}} \ldots \int_{t_{i-1}}^{t} t_{i}^{\beta_{i}} d t_{i} d t_{i-1} \ldots d t_{1} .
$$

It is possible to formulate two main lemmas and a theorem as above also for this case. However, these results can be derived by only making a change of variables as discussed before so we leave out both the formulations and the proofs.

Acknowledgement. The authors would like to thank Professor Ryskul Oinarov, Professor Lars-Erik Persson and the referee for generous advices, which have improved the final version of this paper.

\section{References}

[1] S. Karlin and W.J. Studden, Tchebycheff Systems: with Applications in Analysis and Statistics, Interscience Publishers, New York/London/Sydney, 1966.

[2] M.G. Krejn and A.A. Nudelman, Markov's Moment Problem and Extremum Problems, Nauka, Moscow, 1973. (In Russian).

L.N. Gumilyev Eurasian National University

Munaytpasov st., 5

010008 Astana

Kazakhstan

(E-mail : zamir-a-t@yandex.ru)

Kazakhstan Institute of Management, Economics and Strategic Research

Abai ave., 4

050010 Almaty

Kazakhstan

(E-mail : kalybay@kimep.kz)

(Received: August 2008) 


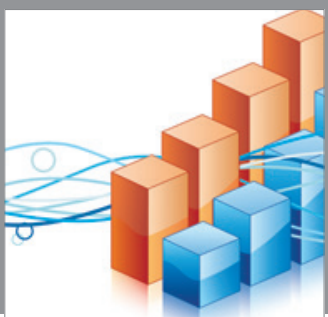

Advances in

Operations Research

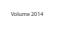

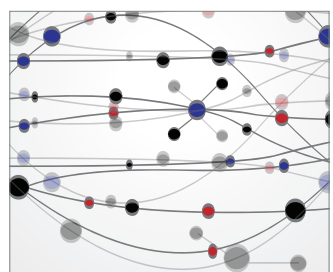

\section{The Scientific} World Journal
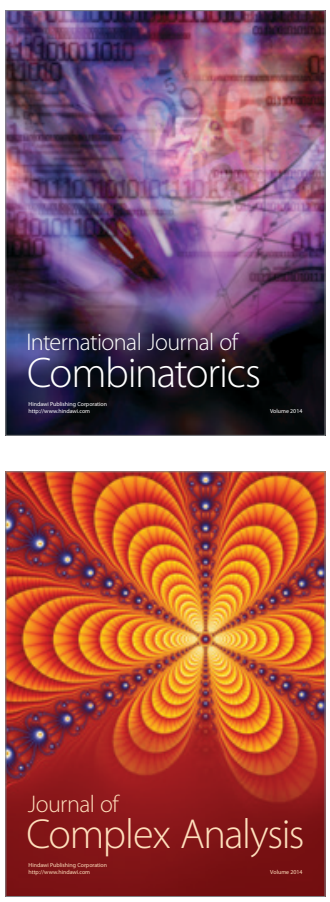

International Journal of

Mathematics and

Mathematical

Sciences
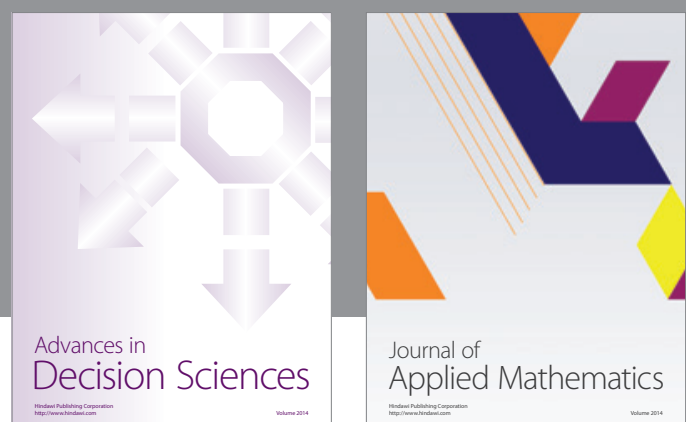

Journal of

Applied Mathematics
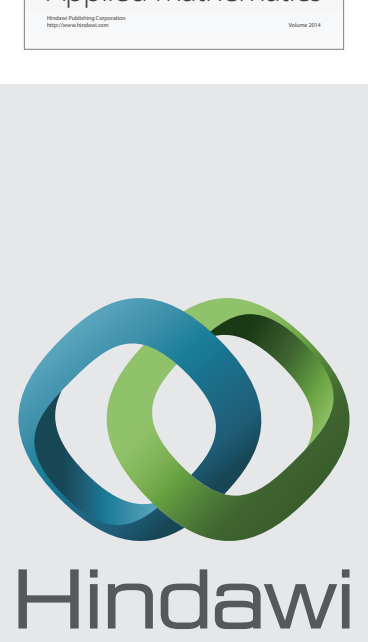

Submit your manuscripts at http://www.hindawi.com
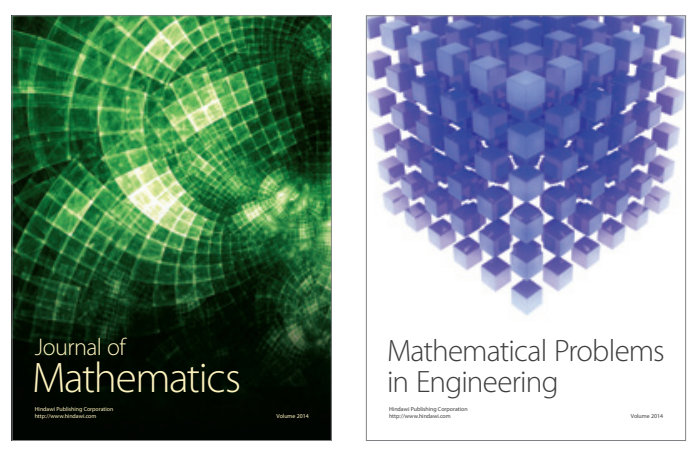

Mathematical Problems in Engineering
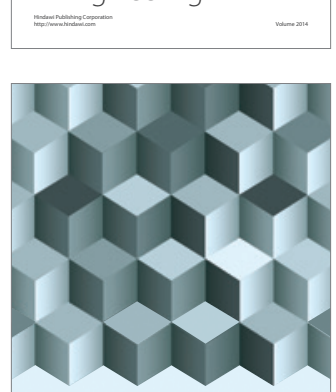

Journal of

Function Spaces
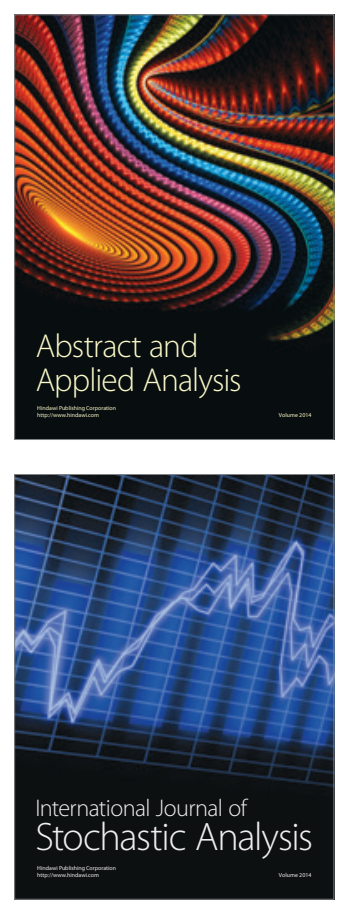

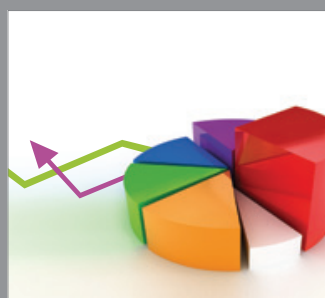

ournal of

Probability and Statistics

Promensencen
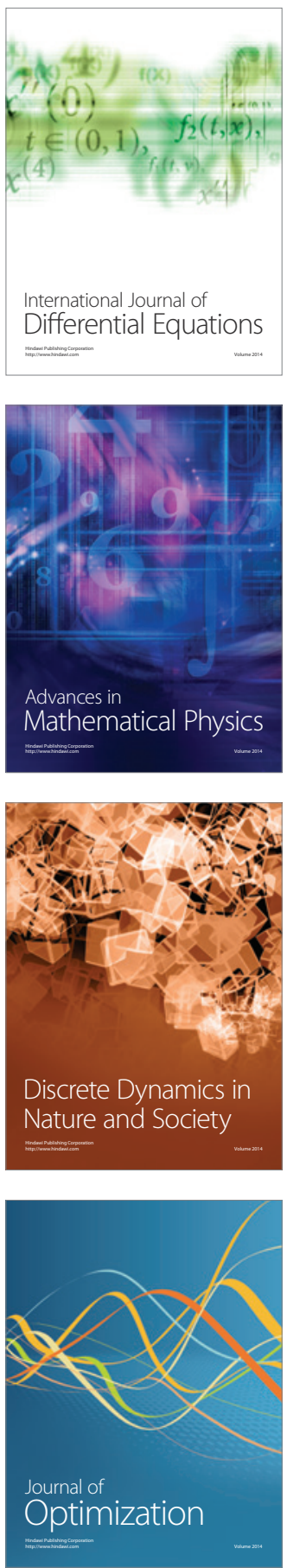